\title{
Hope in Life after Narrative Therapy for Breast Cancer Survivors
}

\author{
A Sajadian ${ }^{1, *}$, L Heydari $^{1}$, A Motaharinasab ${ }^{1}$, L M Raji ${ }^{1}$ \\ ${ }^{1}$ Quality of Life Department, Breast Cancer Research Center, Motamed Cancer \\ Institute, ACECR, Tehran, Iran \\ * Corresponding author: A Sajadian, Quality of Life Department, Breast Cancer \\ Research Center, Motamed Cancer Institute, ACECR, Tehran, Iran. E-mail: \\ assajadi@yahoo.com
}

DOI: $10.21859 /$ mci-supp-96

\section{Keywords:}

Narrative Therapy

Hope

Breast Cancer

Iran

\begin{abstract}
Introduction: Narrative therapy is one of the important approaches to promote quality of life and mental health by increasing hope for female cancer patients. This study aim to evaluate narrative therapy efficacy in increasing hope in breast cancer patients who attended Iranian breast cancer center, Tehran, Iran.

Materials and Methods: We conducted a clinical pretest and posttest study with two experimental groups. All of the participants had already received their primary treatments. Their answered pretest, Herth Hope Index (HHI), was divided to individual therapy and group therapy. 12 patients had individual therapy and 22 patients in 4 groups were treated in group therapy. We had 8 sessions weekly which lasted 100-120 minutes. Narrative therapy was done for two groups. Both groups were re-assessed in order to monitor the changes in hope (posttest) and reported.

Results: This study's statistics showed a mean age of $49.5( \pm 9.0)$, consisting of $54 \%$ educated, $85 \%$ housewives and $77 \%$ married for individual therapy. For group therapy, the mean age was $50.9( \pm 8.9)$, consisting of $82 \%$ educated, $91 \%$ housewives and $82 \%$ married. Over $50 \%$ of patients were in stage II. Data analyzing indicated that age $(\mathrm{P}=0.58)$, marital status $(p=0 / 62)$, occupation $(P=0.53)$, education $(P=0.70)$, staging $(P=0.16)$ and hope pretest $(\mathrm{P}=0.48)$ made no statistically significant difference. Mean scores of hope in individual therapy before and after treatment, respectively are $35.3( \pm 3.2,26.1( \pm 4.5)$, $\mathrm{P}<0.1$; and for group therapy were $27.57( \pm 6 / 7), 29 / 8( \pm 6 / 3) \mathrm{P}=0.0001$. These results indicated an increase in hope during the study for group therapy $(\mathrm{P}<0.0001)$, also there was a significant difference between the two groups $(\mathrm{P}<0.0001)$.

Conclusions: This study showed the effects of narrative therapy specially group therapy in increasing hope in breast cancer patients. It seems group narrative therapies can assist psychological recovery of cancer survivors and promote patients' hope, cope and quality of life.
\end{abstract}

